\section{ralud mental}

Rojas-Carrasco, Karmina Elena; Benjet, Corina; Robles García, Rebeca; Reidl Martínez,

\section{Lucy}

Desarrollo y validación de un instrumento para la evaluación de los subsistemas familiares de pacientes pediátricos hospitalizados por enfermedad crónica (eSisFam)

Salud Mental, vol. 38, núm. 4, julio-agosto, 2015, pp. 259-271

Instituto Nacional de Psiquiatría Ramón de la Fuente Muñiz

Distrito Federal, México
Salud Mental

ISSN: 0185-3325

perezrh@imp.edu.mx

Instituto Nacional de Psiquiatría Ramón

de la Fuente Muñiz

México

Disponible en: http://www.redalyc.org/articulo.oa?id=58241191005

- Cómo citar el artículo

- Número completo

- Más información del artículo

- Página de la revista en redalyc.org 


\title{
Desarrollo y validación de un instrumento para la evaluación de los subsistemas familiares de pacientes pediátricos hospitalizados por enfermedad crónica (eSisFam)
}

\author{
Karmina Elena Rojas-Carrasco,' Corina Benjet, ${ }^{2}$ Rebeca Robles García, ${ }^{2}$ Lucy Reidl Martínez ${ }^{3}$
}

Artículo original

\section{ABSTRACT}

\section{Background}

According to the family systems model, the family consists of three main subsystems: marital, parental and filial, all of which perform specific functions. A chronic pediatric illness presents challenges to these subsystems leading family members to make the necessary adjustments in order to maintain system balance. The evaluation of families in a public hospital setting is hampered by time constraints and limited human resources to carry out a precise evaluation.

\section{Objective}

The aim of this study was to develop and validate a practical instrument for, evaluation of family subsystems during the hospitalization of a pediatric patient with a chronic illness (eSisFam).

\section{Method}

Based on a review of scientific literature and well-known family functioning instruments, a new measure with four sections was developed: the general system and the marital, parental and filial subsystems. This was applied to 312 primary caregivers of chronically ill hospitalized pediatric patients in a public pediatric hospital. Likert-type items with four response options were self-administered with a computer program. Content validity, construct validity and reliability were assessed.

\section{Results}

Content validity was obtained by $80 \%$ agreement between judges. Construct validity was evaluated by exploratory factor analysis, resulting in three factors for the general system (cohesion, communication and adaptation); two for the marital subsystem (affective-sexual relationship and distancing-infidelity); three for the parental subsystem (well-being, shared responsibility and care of siblings), and three for the filial subsystem (well-being, illness knowledge and acceptation-participation). We assessed the internal consistency with Cronbach's alpha coefficients; these were higher than 0.80 in all subscales.

\section{Discussion and conclusion}

The instrument (eSisfam) that was developed is a valid and reliable measure to assess general family system functioning and marital, parental and filial subsystems. This measure could be used in pediatric hospitals for the early detection of family situations that might interfere with child or adolescent medical attention.

Key words: Family assessment, family subsystems, pediatric chronic illness.

\section{RESUMEN}

\section{Antecedentes}

La teoría sistémica de la familia plantea que en ésta existen tres subsistemas: conyugal, parental y filial, los cuales responden, según su particular función, cuando uno de los miembros en edad pediátrica enfrenta alguna enfermedad crónica que amenaza su vida. La evaluación de las familias en un contexto hospitalario institucional presenta distintos desafíos debido al tiempo mínimo necesario para obtener un diagnóstico preciso y por la escasez de recursos humanos preparados para dichas tareas.

\section{Objetivo}

Desarrollar y validar un instrumento para la evaluación de los subsistemas familiares durante la hospitalización a causa de una enfermedad crónica pediátrica (eSisFam).

\section{Método}

A partir de la bibliografía y de instrumentos conocidos de funcionamiento familiar, se conformó uno nuevo de cuatro secciones: sistema general y subsistemas conyugal, parental y filial. Participaron 312 cuidadores primarios de pacientes crónicos internados en un hospital público pediátrico. Los reactivos tipo Likert con cuatro opciones de respuesta, fueron contestados en un programa de cómputo. Se determinó la validez de contenido, de constructo y la confiabilidad.

Hospital de Pediatría CMN S.XXI IMSS. Facultad de Psicología, Universidad Nacional Autónoma de México.

2 Dirección de Investigaciones Epidemiológicas y Psicosociales, Instituto Nacional de Psiquiatría Ramón de la Fuente Muñiz.

Facultad de Psicología, Universidad Nacional Autónoma de México.

Correspondencia: Karmina Elena Rojas-Carrasco. Hospital de Pediatría CMN S.XXI IMSS, consultorio 24, Salud Mental. Cuauhtémoc 330, Doctores, Cuauhtémoc, 06720, México, DF. E-mail: Karmina_rojas@hotmail.com

Recibido primera versión: 4 de julio de 2013. Segunda versión: 21 de julio de 2014. Aceptado: 19 de septiembre de 2014. 


\section{Resultados}

La validez de contenido arrojó más del $80 \%$ de acuerdo interjueces. La validez de constructo por análisis factorial exploratorio identificó tres causas del sistema general (cohesión, comunicación y adaptación), dos del subsistema conyugal (relación afectivo-sexual y distanciamiento-infidelidad), tres del parental (bienestar, responsabilidad compartida y cuidado de los hermanos) y tres del filial (bienestar, conocimiento de la enfermedad y aceptación-participación) con coeficientes alfa de Cronbach superiores a 0.80 .
Discusión y conclusión

El instrumento desarrollado (eSisFam) es válido y confiable para una evaluación general del sistema familiar y de los subsistemas conyugal, parental y filial. La medida podrá ser utilizada en el hospital de pediatría para la detección temprana de situaciones familiares que pudieran interferir con la atención médica del niño o adolescente.

Palabras clave: Evaluación familiar, subsistemas familiares, enfermedad crónica pediátrica.

\section{ANTECEDENTES}

La familia, como sistema básico de formación y bienestar del individuo, es el apoyo principal cuando la salud de alguno de sus miembros se ve afectada. ${ }^{1-3}$ En el contexto de las enfermedades crónicas, la familia sufre y se enferma junto con el paciente ${ }^{4,5}$ pues lo que le sucede a uno de los miembros le afecta de diferentes maneras a los distintos integrantes y subsistemas que la conforman, ${ }^{6,7}$ así como a su funcionamiento y dinámica. ${ }^{8,9}$ Por tanto, que el grupo familiar asumirá cambios para adaptarse a las demandas de la nueva situación, pero al mismo tiempo continuará cumpliendo con sus funciones biopsicosociales. La familia participa más activamente en el proceso de la enfermedad y del tratamiento cuando se trata de niños que en el caso de los pacientes adultos. ${ }^{10}$ Por esta razón, lo que el efecto en la familia puede ser aun mayor, pues el tener un hijo hospitalizado o con una enfermedad que pone en peligro su vida, es particularmente angustiante para los padres y hermanos. ${ }^{11-16}$

Pelechano ${ }^{17}$ refiere que la familia, al enfrentar una enfermedad, debe reestructurarse en cuanto a actividades y papeles; cambios que no siempre son aceptados. Por otra parte, de acuerdo con Lanzarote y Torrado, ${ }^{18}$ una evaluación temprana de la familia puede ayudar a determinar las dificultades y a establecer que resulta fundamental el valorar la adaptación, la cohesión y la comunicación, entre otros aspectos.

Generalmente, durante el proceso de la atención médica el interés se centra en el paciente y su padecimiento, pero pocas veces se toman en cuenta los factores familiares involucrados en el proceso. ${ }^{17} \mathrm{La}$ evaluación de las familias en un contexto hospitalario institucional presenta distintos desafíos, entre los que se encuentran el tiempo limitado para obtener un diagnóstico preciso, la falta de instrumentos prácticos y eficaces para su diagnóstico, y la escasez de recursos humanos preparados para realizar dichas tareas.

Al revisar los instrumentos conocidos para la medición del funcionamiento familiar, ${ }^{19-25}$ se encontró que en general no fueron hechos para personas con familiares que presentan padecimientos crónicos. Debido a esto, la redacción tanto de los reactivos como de las instrucciones no evalúan cambios en la familia debidos a su situación específica por la enfermedad. Ninguno realiza una evaluación conjunta de los subsistemas conyugal (relación entre el cuidador primario y su pareja), parental (las figuras parentales ante la enfermedad) o filial (los hermanos del paciente ante la enfermedad) en una misma familia. La Escala de funcionamiento familiar ante una enfermedad crónica, desarrollada en el 2007 por Alvarado, Barrios y Montero, citados por Montero, ${ }^{26}$ aunque es adecuada para evaluar a la familia con enfermos crónicos, fue validada en pacientes adultos y no se enfoca en los subsistemas familiares ni en sus particulares problemáticas o necesidades de atención.

Por tal motivo, el objetivo del presente estudio fue desarrollar un instrumento de medición del funcionamiento familiar en el sistema general y en los subsistemas conyugal, parental y filial. En cada sección se evalúan aspectos relevantes en la etapa actual de la enfermedad crónica pediátrica durante la hospitalización. El instrumento se aplicó a los cuidadores primarios mediante un programa de cómputo que permitió una rápida y adecuada aplicación y calificación de las respuestas.

\section{MÉTODO}

El estudio se realizó en el hospital de Pediatría del Centro Médico Nacional Siglo XXI del Instituto Mexicano del Seguro Social (IMSS) y fue aprobado por el Comité de ética e investigación del mismo.

\section{Obtención de los reactivos}

Se conformaron reactivos para cada sección a partir de la información reportada en la bibliografía. Para la evaluación del sistema general, se modificaron algunos reactivos que miden cohesión, comunicación y adaptación de las Escalas: clima familiar (FES) de Moss, Moss y Trickette, ${ }^{21}$ funcionamiento familiar (FFSIL) de De la Cuesta, Pérez, Louro y Bayarre $^{27}$ y funcionamiento familiar ante una enfermedad (EFFE) de Montero. ${ }^{26}$ Se escogieron los reactivos que podían ser aplicados en el contexto de las enfermedades crónicas pediátricas. 


\section{Instrumento}

El instrumento se conformó por reactivos que se organizaron en cuatro secciones: una relacionada con el sistema general y tres con los subsistemas conyugal, parental y filial. El sistema general evaluó las dimensiones de cohesión, comunicación y adaptación de la familia en general. El apartado del subsistema conyugal evaluó las dimensiones afectiva-sexual y distanciamiento-infidelidad entre el cuidador primario y su pareja. La sección que evaluó el subsistema parental constó de reactivos que midieron las dimensiones de responsabilidad compartida entre las figuras parentales para la atención de la enfermedad, su bienestar y el cuidado de los hermanos del paciente. Por último, la sección dedicada al subsistema filial, evaluó el conocimiento que los hermanos tienen sobre el padecimiento, su bienestar y participación en las necesidades que surgen durante la atención del hermano enfermo.

El instrumento tipo Likert de cuatro opciones de respuesta (mucho, regular, poco, nada), se aplicó de manera individual a los cuidadores primarios, quienes contestaron en un programa de cómputo especialmente diseñado para que fuese respondido fácilmente sin importar el nivel escolar ni que carecieran de conocimientos en computación.

Cada sección contó con instrucciones específicas y un ejemplo, con el fin de que la persona tomara en cuenta a todos los miembros que componen cada subsistema, dando una respuesta que representara al conjunto.

Es importante señalar que el instrumento se adapta a las familias que no cuentan con algún subsistema. Cada sección puede ser aplicada y calificada por separado de las otras y así obtener una evaluación individual para cada una, ya que no todas las familias se componen de las cuatro secciones evaluadas.

\section{Procedimientos para la validez de contenido}

Acuerdo interjueces expertos. Participaron diez jueces expertos en salud mental: cinco psicólogos clínicos y cinco paidopsiquiatras. Se les pidió que evaluaran los reactivos enlistados por subsistemas, así como sus dimensiones en un programa de cómputo. Igualmente, que señalaran si los reactivos pertenecían o no a la definición operacional (que se les entregó en una hoja impresa) y a la dimensión señalada, de acuerdo con su criterio profesional y experiencia clínica.

Acuerdo intercuidadores primarios. Participaron doce cuidadores primarios (ocho madres, dos padres y dos abuelas) de pacientes del hospital de pediatría. De manera individual fueron leyendo en la computadora los reactivos de cada subsistema y mencionaban al aplicador si tanto éstos como las instrucciones eran claras, comprensibles, difíciles de responder o si no se aplicaban a su situación. También se les pidió que expresaran algún comentario o sugerencia que sirviera para mejorar las preguntas.

\section{Procedimiento para la validez de constructo}

En un primer paso, se solicitó la participación voluntaria de 312 cuidadores primarios de pacientes pediátricos crónicos hospitalizados en las áreas de preescolares, escolares y adolescentes de un hospital de tercer nivel de atención. Los que aceptaron participar firmaron la carta de consentimiento informado. Los cuidadores primarios podían ser la madre, el padre $u$ otro familiar o conocido que estuviera la mayor parte del tiempo con el paciente en el hospital. La aplicación de los 210 reactivos iniciales se realizó en computadoras portátiles.

Cuadro 1. Características de las familias participantes

\begin{tabular}{|c|c|c|c|c|c|}
\hline & $\mathrm{N}$ & $\%$ & & $\mathrm{~N}$ & $\%$ \\
\hline Edad del paciente & & & Procedencia de la familia & & \\
\hline Preescolar & 76 & 24 & DF o Estado de México & 412 & 45 \\
\hline Escolar & 109 & 35 & Otro estado del interior & 170 & 55 \\
\hline Adolescente & 127 & 41 & & & \\
\hline Parentesco del cuidador primario con el paciente & & & Número de hermanos del paciente & & \\
\hline Madre & 271 & 87 & Ninguno & 46 & 15 \\
\hline Padre & 26 & 8 & Uno & 130 & 42 \\
\hline Abuela materna & 7 & 2 & Dos & 99 & 32 \\
\hline Otros (primos, tíos, cuñados, madrastra, etc.) & 8 & 3 & Tres o más & 37 & 11 \\
\hline Tipo de familia & & & Tipo de enfermedad del paciente & & \\
\hline Nuclear biparental & 174 & 56 & Oncológica o hematológica* & 105 & 34 \\
\hline Extensa biparental & 49 & 16 & Neurológica o neurocirugía & 68 & 22 \\
\hline Nuclear uniparental & 42 & 13 & Nefrológica o urológica & 39 & 12 \\
\hline Extensa uniparental & 40 & 13 & $\begin{array}{l}\text { Otras (reumatología, infectología, neumología, cardiología, } \\
\text { maxilo-facial, etc.) }\end{array}$ & 69 & 22 \\
\hline Compuesta & 7 & 2 & & & \\
\hline
\end{tabular}

*Incluyen leucemias y otros padecimientos hematológicos. 
Cuadro 2. Sistema General. Se extrajeron 3 factores con valores Eigen de 1 o mayores

\begin{tabular}{|c|c|c|c|}
\hline \multirow[b]{2}{*}{ Reactivos del Subsistema General } & \multicolumn{3}{|c|}{ Factores } \\
\hline & 1 & 2 & 3 \\
\hline \multicolumn{4}{|l|}{ En esta etapa de la enfermedad, en nuestra familia...* } \\
\hline G54...nos apoyamos unos a otros mientras el paciente está en el hospital & .809 & & \\
\hline G55...se toman decisiones en conjunto para cosas importantes de la enfermedad & .726 & & \\
\hline G37...hay unión para enfrentar la enfermedad & .707 & & \\
\hline G44...se apoya a la persona que más cuida al paciente & .698 & & \\
\hline G53...cuando alguno tiene un problema, otros se ocupan de ayudarlo & .695 & & \\
\hline G52...en común acuerdo, cubrimos las necesidades del paciente & .691 & & \\
\hline G47...cuando surge algún problema por la enfermedad, proponemos soluciones & .669 & & \\
\hline G49...somos organizados para visitar al enfermo en el hospital & .661 & & \\
\hline G2...contamos con el apoyo de nuestros familiares & .661 & & \\
\hline G20...cada integrante asume la responsabilidad que le corresponde & .659 & & \\
\hline G46...aunque no estemos presentes, nos mantenemos interesados en la situación del paciente & .641 & & \\
\hline G10...planeamos actividades para cubrir las necesidades de la familia & .637 & & \\
\hline G60...es importante mantenernos juntos en este momento & .592 & & \\
\hline G7...a pesar de la enfermedad, predomina la armonía & .584 & & \\
\hline G33...planeamos actividades para cubrir las necesidades del paciente & .584 & & \\
\hline G19...el cuidador principal se mantiene en contacto con otros familiares & .575 & & \\
\hline G48...es importante mantenernos informados sobre la enfermedad & .559 & & \\
\hline G27...las necesidades del cuidador principal también nos importan & .544 & & \\
\hline G42...hablamos sobre las necesidades del paciente & .538 & & \\
\hline G29...pensamos que la responsabilidad del paciente es de todos & .478 & & \\
\hline G8...platicamos sobre lo que sentimos por la enfermedad & & 690 & \\
\hline G31...hablamos abiertamente de lo que nos está pasando & & .666 & \\
\hline G13...podemos hablar unos con otros acerca de la tristeza que sentimos & & 650 & \\
\hline G30...se permite llorar cuando estamos tristes por el paciente & & 632 & \\
\hline G22...podemos conversar sobre la enfermedad y su tratamiento sin temor & & .621 & \\
\hline G35... platicamos sobre lo que puede suceder con el paciente en el futuro & & .614 & \\
\hline G16...podemos expresar la tristeza con llanto & & .592 & \\
\hline G4...podemos hablar abiertamente sobre lo que pensamos de la enfermedad & & .482 & \\
\hline G3...mostramos tanto los sentimientos positivos como los negativos & & .470 & \\
\hline G12...cuando alguien se enoja por la situación del paciente, puede expresarlo & & .402 & \\
\hline G40...andamos "como en otro planeta" & & & .727 \\
\hline G45...nos ha sido difícil hacer cosas diferentes o nuevas debido a la enfermedad & & & .642 \\
\hline G18...nos ha sido difícil aceptar que esto nos esté pasando & & & .614 \\
\hline G43...es difícil llegar a acuerdos con respecto a la enfermedad & & & .598 \\
\hline G28...nos cuesta trabajo cambiar nuestros hábitos o rutinas & & & .531 \\
\hline G23...es difícil cumplir con las reglas del hospital & & & .520 \\
\hline G50...tratamos de hacer la vida lo más normal posible & & & .491 \\
\hline G62...nos ha sido difícil dejar de hacer cosas que antes hacíamos & & & .489 \\
\hline G41... a pesar de la situación difícil, nos damos tiempo para distraernos & & & .462 \\
\hline G36...peleamos o discutimos por causa de esta situación & & & .445 \\
\hline G59...podemos hablar de la enfermedad sin gritar, desesperarnos o llorar & & & .413 \\
\hline G24...hemos podido organizar el hogar en torno a la enfermedad & & & .410 \\
\hline
\end{tabular}

\section{Análisis de datos}

Para la validez de contenido se obtuvo el porcentaje de acuerdo interjueces. Para la validez de constructo se evaluó el poder discriminativo de los reactivos por medio de la comparación de las medias con la prueba t para muestras independientes (entre los cuidadores que respondieron con puntajes bajos vs los que respondieron con puntajes altos). Se realizaron análisis factoriales exploratorios para cada sección. Se obtuvieron los coeficientes alfa de Cronbach por cada factor del sistema general y de los subsistemas, así como de cada sección en total. Para el análisis de los datos, se utilizó el paquete SPSS versión 20.

\section{RESULTADOS}

En el cuadro 1 se muestran las características de las familias participantes, donde se observa que el $87 \%$ de los cuidadores primarios son las madres, aproximadamente la mitad 
Cuadro 3. Subsistema Conyugal. Se extrajeron 2 factores con valores Eigen de 1 o mayores

\begin{tabular}{|c|c|c|}
\hline \multirow[b]{2}{*}{ Reactivos del Subsistema Conyugal } & \multicolumn{2}{|c|}{ Factores } \\
\hline & 1 & 2 \\
\hline \multicolumn{3}{|l|}{ En esta etapa de la enfermedad, como pareja...* } \\
\hline C19...estamos interesados en nuestra relación sexual & .809 & \\
\hline C14...podemos tener satisfacción sexual & .787 & \\
\hline C18...tratamos de dedicarnos tiempo uno al otro & .780 & \\
\hline C5...tratamos de conservar nuestro espacio íntimo & .762 & \\
\hline C8...podemos disfrutarnos mutuamente en este momento & .758 & \\
\hline C9... demostramos el amor que sentimos hacia el otro & .716 & \\
\hline C24...podemos sentir pasión el uno por el otro & .707 & \\
\hline C3...nuestra sexualidad es importante & .679 & \\
\hline C31...mostramos interés sexual por el otro & .667 & \\
\hline C32...somos atractivos para el otro & .661 & \\
\hline C1...nos queda tiempo para convivir & .624 & \\
\hline C21...nos damos un tiempo para salir solos & .593 & \\
\hline C4...comprendemos lo que siente el otro & .587 & \\
\hline C7...evitamos el tema de nuestra sexualidad & .514 & \\
\hline C22...ha bajado la calidad de nuestras relaciones sexuales & .470 & \\
\hline C28...hemos disminuido nuestras relaciones sexuales & .468 & \\
\hline C29...sentimos culpa por disfrutar nuestra relación sexual & .400 & \\
\hline C23...hemos estado al punto del divorcio a causa de la enfermedad & & .702 \\
\hline C34...nos ha interesado otra persona & & .690 \\
\hline C17...ha habido infidelidad & & .666 \\
\hline C $30 . .$. uno de los dos dice que la enfermedad es un pretexto para engañar al otro & & .660 \\
\hline C20...nos hemos alejado emocionalmente el uno del otro & & .602 \\
\hline C6...la enfermedad nos provocó la separación & & .572 \\
\hline C33...si alguno es infiel piensa que el otro hace lo mismo & & .556 \\
\hline C12...hemos mostrado interés sexual por otra persona & & .543 \\
\hline C15...uno de nosotros se siente desplazado por el paciente & & .494 \\
\hline C25...estamos alejados porque uno está en el hospital y el otro trabajando & & .415 \\
\hline
\end{tabular}

*Frase que aparece en cada uno de los reactivos del subsistema conyugal.

son del DF o Estado de México, y la otra mitad son foráneos. Una tercera parte son pacientes oncológicos o hematológicos.

\section{Validez de contenido}

Todos los reactivos fueron aceptados por los jueces en sus respectivas dimensiones y se tomaron en cuenta algunas observaciones, como cambiar una palabra por otra o hacer más sencilla la redacción. Se obtuvo un porcentaje de acuerdo interjueces por arriba del $80 \%$ en el total de los reactivos por cada dimensión.

\section{Validez de constructo}

La prueba t mostró que todos los reactivos del sistema general y del subsistema conyugal discriminaron entre los puntajes altos y bajos de la muestra. En el subsistema parental sólo un reactivo no discriminó y en el filial fueron tres los que no discriminaron; por lo tanto se eliminaron.

En los cuadros 2 al 5 se muestran las cargas factoriales obtenidas en el análisis exploratorio de los reactivos del sistema general (cohesión, comunicación y adaptación; éstos explican el 37\% de la varianza) y de los subsistemas conyugal (relación afectivo-sexual y distanciamiento-infidelidad que explican el $42.5 \%$ de la varianza), parental (bienestar, responsabilidad compartida y cuidado de los hermanos, explican el $48 \%$ de la varianza), así como filial (bienestar, conocimiento de la enfermedad y aceptación-participación, que explican el $40 \%$ de la varianza). Se eliminaron los reactivos con cargas factoriales menores a 0.40 y aquellos que cargaban alto en dos o más factores. Después del análisis factorial quedaron un total de 144 reactivos de los 210 iniciales, los cuales conforman el instrumento de evaluación del sistema familiar que nominamos eSisFam (anexo 1).

El cuadro 6 presenta el sistema general y los subsistemas, con el número de reactivos finales, así como las definiciones operacionales correspondientes a cada factor obtenido.

\section{Confiabilidad de consistencia interna}

Se obtuvieron los coeficientes Alfa de Cronbach para cada uno de los factores así como para el total de cada sección. Se obtuvieron valores por arriba del 0.80 (cuadro 7). 
Cuadro 4. Subsistema Parental. Se extrajeron 3 factores con valores Eigen de 1 o mayores

\begin{tabular}{|c|c|c|c|}
\hline \multirow[b]{2}{*}{ Reactivos del Subsistema Parental } & \multicolumn{3}{|c|}{ Factores } \\
\hline & 1 & 2 & 3 \\
\hline \multicolumn{4}{|l|}{ En esta etapa de la enfermedad, como padres... ${ }^{*}$} \\
\hline P41...estamos deprimidos & .788 & & \\
\hline P47...nos encontramos desesperados & .786 & & \\
\hline P32...nos sentimos frustrados ante esta enfermedad & .721 & & \\
\hline P39...estamos desanimados con la situación actual de nuestro hijo & .715 & & \\
\hline P43...estamos ansiosos & .683 & & \\
\hline P45...estamos preocupados & .671 & & \\
\hline P3...hemos tenido problemas para dormir & .650 & & \\
\hline P28...nos sentimos culpables de la enfermedad de nuestro hijo & .617 & & \\
\hline P6...hemos descuidado nuestra salud por atender al paciente & .610 & & \\
\hline P34... hemos padecido dolores de cabeza, de estómago, del cuerpo u otro malestar & .594 & & \\
\hline P35...estamos enojados por esta difícil situación & .585 & & \\
\hline P37...nos sentimos impotentes ante la enfermedad de nuestro hijo & .576 & & \\
\hline P48...uno o ambos ha enfermado a causa de esta situación & .570 & & \\
\hline P9...hemos alterado nuestra alimentación & .562 & & \\
\hline P50...uno o ambos ya estamos cansados o fatigados por la situación & .530 & & \\
\hline P14...nos hemos enfermado con frecuencia & .430 & & \\
\hline P19... ambos estamos al tanto del enfermo y su padecimiento & & .793 & \\
\hline P1...compartimos la responsabilidad de la enfermedad & & .787 & \\
\hline P25...nos brindamos apoyo moral & & .763 & \\
\hline P7...sentimos que la responsabilidad del hogar es de los dos & & .754 & \\
\hline P30...aunque uno esté ausente del hospital, se mantiene al tanto de la situación & & .752 & \\
\hline P27...nos apoyamos en las decisiones sobre procedimientos médicos & & .749 & \\
\hline P13...organizamos las actividades del hogar en mutuo acuerdo & & .682 & \\
\hline P24...nos brindamos apoyo económico & & .675 & \\
\hline P15...nos apoyamos en las tareas de la casa & & .640 & \\
\hline P21...estamos al pendiente de las indicaciones médicas & & .526 & \\
\hline P18...uno lleva más la carga de la situación que el otro & & .524 & \\
\hline P23...estamos informados de lo que sucede con el enfermo en el hospital & & .519 & \\
\hline P53...entendemos el estado de ánimo de nuestros hijos sanos & & & .823 \\
\hline P42...escuchamos a nuestros otros hijos cuando quieren conversar & & & .762 \\
\hline P20...satisfacemos las necesidades de los otros hijos & & & .716 \\
\hline P51...sabemos lo que sienten nuestros hijos por su hermano enfermo & & & .715 \\
\hline P40...tomamos en cuenta la opinión de los otros hijos & & & .665 \\
\hline P44...demostramos cariño a nuestros otros hijos & & & .657 \\
\hline P33...creemos que la atención que ponemos a los otros hijos es adecuada & & & .505 \\
\hline
\end{tabular}

*Frase que aparece en cada uno de los reactivos del subsistema parental.

\section{DISCUSIÓN Y CONCLUSIÓN}

El contar con instrumentos específicos para la evaluación de los subsistemas familiares, en el contexto de las enfermedades crónicas pediátricas, es necesario para comprender con mayor profundidad situaciones intrafamiliares que se alteran al enfrentar un padecimiento. ${ }^{6-9}$ Por ello se requiere de la evaluación oportuna de los servicios de salud mental como parte integral de la atención del paciente hospitalizado. ${ }^{28}$

El instrumento desarrollado proporciona una valoración del sistema general donde se evalúa la cohesión, comunicación y adaptación de la familia a la situación actual del padecimiento. Estos factores son evaluados en la mayoría de los instrumentos de funcionamiento familiar, ${ }^{19-27}$ pero no fueron desarrollados específicamente para situaciones de enfermedades crónicas pediátricas.
Debido a que los subsistemas familiares se ven seriamente afectados ante la enfermedad ${ }^{6,7}$ (por ejemplo, conflictos en la relación de pareja de los padres, ${ }^{29,30}$ cuando se deja la mayor parte de la responsabilidad a uno de ellos ${ }^{31,32}$ o cuando los hermanos se sienten desplazados o abandonados), ${ }^{33-35}$ el nuevo instrumento proporciona una valoración del subsistema conyugal (relación afectiva-sexual y distanciamiento-infidelidad), del subsistema parental (bienestar, responsabilidad compartida y cuidado de los hermanos) y del subsistema filial (bienestar, conocimiento de la enfermedad y aceptación-participación). La evaluación de estos tres en los factores propuestos y validados es la nueva aportación de este trabajo al campo del diagnóstico familiar.

Es pertinente mencionar que el utilizar la computadora para la aplicación de instrumentos es un recurso de apoyo para el diagnóstico temprano en hospitales públicos de alta 
Cuadro 5. Subsistema Filial. Se extrajeron 3 factores con valores Eigen de 1 o mayores

\begin{tabular}{|c|c|c|c|}
\hline \multirow[b]{2}{*}{ Reactivos del Subsistema Filial } & \multicolumn{3}{|c|}{ Factores } \\
\hline & 1 & 2 & 3 \\
\hline En esta etapa de la enfermedad, los hermanos del paciente...* & & & \\
\hline F38...han desarrollado miedos & .725 & & \\
\hline F36...se han mostrado desanimados & .711 & & \\
\hline F37... han presentado problemas en su alimentación & .706 & & \\
\hline F40...se han sentido solos porque los padres están en el hospital & .672 & & \\
\hline F18... han padecido dolores de cabeza, de estómago, del cuerpo u otro malestar & .647 & & \\
\hline F17... han mostrado enojo por la situación de su hermano enfermo & .611 & & \\
\hline F20...han presentado insomnio, pesadillas $u$ otros problemas para dormir & 611 & & \\
\hline F3...han tenido cambios bruscos en su estado de ánimo & .602 & & \\
\hline F14...preguntan como reclamo "¿por qué mi hermano tiene esta enfermedad?" & .568 & & \\
\hline F52... han visto afectadas algunas de sus actividades a causa de la enfermedad & .562 & & \\
\hline F24...se les dificulta quedarse al cuidado de otras personas & .527 & & \\
\hline F8...han presentado problemas de conducta & .516 & & \\
\hline F15...han bajado su rendimiento escolar & .509 & & \\
\hline F6...se han enfermado con frecuencia & .509 & & \\
\hline F34...han expresado sentir soledad por la ausencia de su hermano & .509 & & \\
\hline F30...tienen miedo de enfermar como su hermano & .500 & & \\
\hline F54...han expresado sentir culpa por estar sanos & .472 & & \\
\hline F46...se han aislado de los demás & .463 & & \\
\hline F22...se han sentido culpables por la enfermedad de su hermano & .429 & & \\
\hline F31...tienen información verdadera de la enfermedad & & .803 & \\
\hline F21...tienen información clara de la enfermedad & & .796 & \\
\hline F25...les explicamos en qué consiste el tratamiento a manera que lo entiendan & & .767 & \\
\hline F19...saben en qué consiste esta enfermedad & & .740 & \\
\hline F1...están enterados de la enfermedad de su hermano & & .739 & \\
\hline F23...comprenden la situación actual del paciente & & .727 & \\
\hline F35...les explicamos algunos procedimientos médicos de manera que los entiendan & & .718 & \\
\hline F4...saben de los cuidados que debe tener su hermano enfermo & & .671 & \\
\hline F7...les decimos cómo está su hermano enfermo cuando está en el hospital & & .646 & \\
\hline F12...han platicado con el paciente sobre su enfermedad & & .558 & \\
\hline F33...parecen entender cuando les explicamos esta situación & & .545 & \\
\hline F53...muestran cariño hacia su hermano enfermo & & & .748 \\
\hline F41...se muestran comprensivos con el paciente & & & .658 \\
\hline F43...participan en el cuidado de su hermano enfermo cuando está en casa & & & .642 \\
\hline F49...cuidan a su hermano enfermo de caídas, golpes o algo que pudiera lastimarlo & & & .633 \\
\hline F51 ...animan al paciente a cuidar su salud & & & .627 \\
\hline F42...muestran rechazo por su hermano enfermo & & & .588 \\
\hline F39...le hablan por teléfono o le envían una carta al paciente cuando está en el hospital & & & .503 \\
\hline F27...cooperan en las actividades del hogar como cada uno puede & & & .486 \\
\hline F29... desean visitar a su hermano en el hospital & & & .473 \\
\hline F10...juegan o conviven con su hermano enfermo & & & .470 \\
\hline
\end{tabular}

*Frase que aparece en cada uno de los reactivos del subsistema filial.

concentración, donde generalmente los recursos para la evaluación de factores psicosociales de la enfermedad son limitados. ${ }^{33}$ Por otra parte, con la creciente utilización de los expedientes electrónicos, en el futuro se tendrá la posibilidad de contar con un perfil o diagnóstico familiar de cada paciente de manera rápida y eficiente. Además, esta forma de aplicación ayudó a que los participantes se mostraran interesados y motivados por responder de forma novedosa y dinámica.

El instrumento desarrollado es válido y confiable para una evaluación general del sistema familiar y de los sub- sistemas. La medida podrá ser utilizada en el hospital de pediatría para la detección temprana de situaciones familiares que pudieran interferir en la atención médica del niño o adolescente.

\section{Limitaciones}

La limitación principal de este trabajo es el no haber aplicado cada subsistema a los diferentes miembros involucrados. Sería ideal que los hermanos del paciente respondieran a las preguntas del subsistema filial, que la pareja del cuidador 
Rojas-Carrasco et al.

Cuadro 6. Número de reactivos para cada subsistema y definiciones operacionales de los factores obtenidos para el eSisFam

\begin{tabular}{|c|c|c|c|}
\hline Sección & Factores & $\begin{array}{l}\text { Núm. de } \\
\text { reactivos }\end{array}$ & Definición operacional \\
\hline \multirow[t]{3}{*}{ General (42) } & Cohesión & 20 & $\begin{array}{l}\text { Evalúa la unión entre los miembros de la familia, el apoyo que dan al cuidador primario } \\
\text { mientras está con el paciente en el hospital. Actitud de colaboración de los integrantes } \\
\text { como si la situación de la enfermedad tuviera que ver con todos. Acuerdos en conjunto } \\
\text { para solucionar las demandas del padecimiento en la etapa actual del mismo. }\end{array}$ \\
\hline & Comunicación & 10 & $\begin{array}{l}\text { Evalúa la expresión de pensamientos y sentimientos de los integrantes sobre la enferme- } \\
\text { dad del paciente en esta etapa de la enfermedad. }\end{array}$ \\
\hline & Adaptación & 12 & $\begin{array}{l}\text { Evalúa la capacidad de la familia para modificar sus hábitos y costumbres, organizarse, } \\
\text { cumplir con las reglas del hospital y tratar de continuar con su vida cotidiana normal } \\
\text { durante la actual etapa de la enfermedad. }\end{array}$ \\
\hline \multirow[t]{2}{*}{ Conyugal (27) } & Relación afectiva-sexual & 17 & $\begin{array}{l}\text { Evalúa las demostraciones de atención, cariño y comprensión, así como aspectos de la } \\
\text { relación sexual entre el cuidador primario y su pareja (aunque ésta no sea el padre o } \\
\text { figura paterna del paciente) durante la actual etapa de la enfermedad. }\end{array}$ \\
\hline & $\begin{array}{l}\text { Distanciamiento-infide- } \\
\text { lidad }\end{array}$ & 10 & $\begin{array}{l}\text { Evalúa las actitudes de distanciamiento e infidelidad entre el cuidador primario y su } \\
\text { pareja (aunque ésta no sea el padre o figura paterna del paciente) durante la actual } \\
\text { etapa de la enfermedad. }\end{array}$ \\
\hline \multirow[t]{3}{*}{ Parental (35) } & Bienestar & 16 & $\begin{array}{l}\text { Evalúa la salud física y emocional de la pareja parental en la etapa actual de la enfer- } \\
\text { medad. }\end{array}$ \\
\hline & $\begin{array}{l}\text { Responsabilidad com- } \\
\text { partida }\end{array}$ & 12 & $\begin{array}{l}\text { Evalúa qué tanto la pareja parental comparte la responsabilidad del hijo enfermo y del } \\
\text { hogar durante la actual etapa del padecimiento. }\end{array}$ \\
\hline & $\begin{array}{l}\text { Cuidado de los herma- } \\
\text { nos }\end{array}$ & 7 & $\begin{array}{l}\text { Evalúa el cuidado o abandono de los hermanos mientras las figuras paternas atienden } \\
\text { al paciente, en la etapa actual de la enfermedad. }\end{array}$ \\
\hline \multirow[t]{3}{*}{ Filial (40) } & Bienestar & 19 & $\begin{array}{l}\text { Evalúa la salud física y emocional de los hermanos del paciente así como cambios } \\
\text { conductuales y escolares, en la etapa actual de la enfermedad. }\end{array}$ \\
\hline & $\begin{array}{l}\text { Conocimiento de la } \\
\text { enfermedad }\end{array}$ & 11 & $\begin{array}{l}\text { Evalúa si los hermanos del paciente tienen información clara, verídica y comprensible } \\
\text { para su edad, de la enfermedad, de los cuidados que requiere el paciente y del trata- } \\
\text { miento o procedimientos médicos durante la etapa actual de la enfermedad. }\end{array}$ \\
\hline & Aceptación-participación & 10 & $\begin{array}{l}\text { Evalúa el cariño y aceptación de los hermanos hacia el paciente, la participación en su } \\
\text { cuidado y en el hogar durante la actual etapa de la enfermedad. }\end{array}$ \\
\hline
\end{tabular}

Cuadro 7. Coeficientes Alpha de Cronbach para los factores extraídos y en total para cada sección

\begin{tabular}{|c|c|c|c|c|}
\hline \multirow[b]{2}{*}{ Subsistema } & \multirow[b]{2}{*}{ Factores } & \multirow{2}{*}{$\begin{array}{l}\text { Núm. de } \\
\text { reactivos }\end{array}$} & \multicolumn{2}{|c|}{ Coef. Alpha de Cronbach } \\
\hline & & & Por factor & Por subsistema \\
\hline \multirow[t]{3}{*}{ General } & Cohesión & 20 & 0.936 & 0.930 \\
\hline & Comunicación & 10 & 0.855 & \\
\hline & Adaptación & 12 & 0.812 & \\
\hline \multirow[t]{2}{*}{ Conyugal } & Relación afectiva-sexual & 17 & 0.929 & 0.926 \\
\hline & Distanciamiento-infidelidad & 10 & 0.812 & \\
\hline \multirow[t]{3}{*}{ Parental } & Bienestar & 16 & 0.914 & 0.920 \\
\hline & Responsabilidad compartida & 12 & 0.903 & \\
\hline & Cuidado de los hermanos & 7 & 0.847 & \\
\hline \multirow[t]{3}{*}{ Filial } & Bienestar & 19 & 0.895 & 0.898 \\
\hline & Conocimiento de la enfermedad & 11 & 0.908 & \\
\hline & Aceptación-participación & 10 & 0.849 & \\
\hline
\end{tabular}

principal respondiera al subsistema conyugal, y que la otra figura parental respondiera al subsistema parental. Lo anterior, debido a que la validación que se presenta se basa exclusivamente en la percepción del cuidador primario sobre los subsistemas familiares, lo cual puede reflejar un sesgo muy importante al expresar sus deseos y no la realidad. Debido a que casi la mitad de las familias provienen de otros Estados de la República, y el padre (casi siempre) trabaja de tiempo com- pleto, es poco factible en el contexto de hospitales públicos de tercer nivel contar con los demás miembros de la familia.

\section{Financiamiento}

Ninguno.

\section{Conflicto de intereses}

Los autores declararon no tener conflicto de intereses. 


\section{Agradecimientos}

A la doctora Shoshana Berezon-Gorn del Istituto Nacional de Psiquiatría Ramón de la Fuente Muñiz y al doctor Javier Aguilar-Hernández de la Facultad de Psicología de la UNAM, por sus valiosas asesorías metodológicas. A los psicólogos clínicos y paidopsiquiatras que participaron como jueces.

\section{REFERENCIAS}

1. Foley B, Barakat LP, Herman-Liu A, Radcliffe J et al. The impact of childhood hypothalamic/chiasmatic brain tumors in child adjustment and family functioning. Children's Health Care 2000;29(3):209-223.

2. Patterson JM, Holm KE, Gurney JG. The impact of childhood cancer on the family: A qualitative analysis of strains, resource and coping behaviors. Psycho-Oncology 2004;13:390-407.

3. Fanos J, Fahrner K, Jelveh M, King R et al. The sibling center: A pilot program for siblings of children and adolescents with a serious medical condition. J Pediatrics 2005;143:831-835.

4. Van Dyck PC, Kogan MD, McPherson MG, Weissman GR et al. Prevalence and characteristic of children with special health care needs (reprinted). Archives Child Health, Pediatrics Adolescent Medicine 2004;158:884-890.

5. McClellan CB, Cohen LL. Family functioning in children with chronic illness compared with healthy controls: a critical review. J Pediatrics 2007;150:221-223.

6. Minuchin S. Familias y terapia familiar. Argentina: Editorial Gedisa; 1974.

7. Williams PD, Williams AR, Graff JC, Hanson S et al. Interrelationships among variables affecting well siblings and mothers in families with a chronic illness or disability. J Behavioral Medicine 2002;25:411424.

8. Ramos B, Aldereguía J. Medicina social y salud pública en Cuba. Ciudad de la Habana: Editorial Pueblo y Educación; 1990.

9. Roca MA, Pérez M. Apoyo social. Su significación para la salud humana. La Habana Cuba: Editorial Félix Varela; 2000.

10. Taylor SE. Psicología de la salud. México: McGraw-Hill/Interamericana Editores; 2007.

11. Cordaro G, Veneroni L, Massimino M, Clerici CA. Assessing psychological adjustment in siblings of children with cancer: parents' perspectives. Cancer Nursing 2012;35(1):42-50.

12. Ten Hoedt AE, Maurice-Stam H, Boelen CCA, Rubio-Gozalbo ME et al. Parenting a child with phenylketonuria or galactosemia: implications for health-related quality of life. J Inheritable Metabolic Disease 2011;34:391-398.

13. Kao B, Plante W, Lobato D. The use of the Impact on Sibling Scale with families of children with chronic illness and developmental disability. Child: Care, Health Development 2009;35(4):505-509.

14. Williams PD, Ridder EL, Setter RD, Liebergen A. Pediatric chronic illness (cancer, cystic fibrosis) effects on well siblings: parents' voices. Comprehensive Pediatric Nursing 2009;32:94-113.

15. Hopia H, Tomlinson PS, Paavilainen E, Astedt-Kurki P. Child in hospital: family experiences and expectations of how nurses can promote family health. J Clinical Nursing 2005;14:212-222.
16. Garro A, Thurman SK, Kerwin ME, Ducette JP. Parent/caregiver stress during pediatric hospitalization for chronic feeding problems. J Pediatric Nurcing 2005;20:268-275.

17. Pelechano V. Calidad de vida, familia y afrontamiento en la enfermedad física crónica: Datos y sugerencias para un modelo. En: Buendía J (ed.). Familia y psicología de la salud. Madrid: Pirámide; 1999.

18. Lanzarote MD, Torrado E. Orientación y psicoterapia familiar con niños y adolescentes con enfermedad crónica. Apuntes Psicología 2009;27(23):457-471.

19. Epstein NB, Baldwin LM, Bishop DS. The McMaster family assessment device. J Marital Family Therapy 1983;9:171-180.

20. García-Mendez M, Rivera-Aragón S, Reyes-Lagunes I, Díaz-Loving R. Construcción de una escala de funcionamiento familiar. Revista Iberoamericana Diagnóstico Evaluación Psicológica 2006;22(2):91-110.

21. Moos RH, Moos BS, Trickett E. Escalas de clima social. Madrid: Tea Ediciones; 1995.

22. Olson DH, Russell C, Sprenkle DH. Circumplex model of marital and family sistems II: Empirical studies and clinical intervention. Advances Family Intervention, Assessment Theory 1989;1:129-176.

23. Palomar J. Funcionamiento familiar y calidad de vida. Tesis de doctorado en psicología. Facultad de Psicología. Universidad Nacional Autónoma de México. México, DF; 1998.

24. Smilkstein G. The family APGAR: A proposal for a family function test and its used by physicians. J Family Practice 1978;6:12-31.

25. Villatoro JA, Andrade P, Fleiz C, Medina-Mora ME et al. La relación padres-hijos: una escala para evaluar el ambiente familiar de los adolescentes. Salud Mental 1997;20:2-27.

26. Montero Pardo X. Enfermedad crónica y familia. Tesis para obtener el grado de maestra en psicología. Facultad de Psicología, Universidad Nacional Autónoma de México. México, DF; 2009.

27. De la Cuesta D, Pérez E, Louro I, Bayarre H. Un instrumento de funcionamiento familiar para el médico de la familia. Revista Cubana Medicina General Integral 1996;12(1):24-3:24-31.

28. Sauceda JM. Lineamientos para evaluar la vida en familia. Revista Médica IMSS 1995;33:249-256.

29. Backalarz. La familia frente a la enfermedad crónica. Charla brindada en el Seminario de Actualización sobre Neurofibromatosis. 2007. Recuperado febrero, 2012 http://www.aanf.org.ar/documento/13.pdf

30. Morrod D. Make or break - who cares for couples when their children are sick? Sexual Relationship Therapy 2004;19(3):247-263.

31. Nagy S, Ungerer J. The adaptation of mothers and fathers to children with cystic fibrosis: a comparison. Children's Healt Care 1990;9(3):147154.

32. Rolland JS. Familias, enfermedad y discapacidad. Una propuesta desde la terapia sistémica. Barcelona: Gedisa; 2000.

33. Sauceda JM, Morales ME, Anaya A, Escalante P et al. Aportaciones de la psiquiatría de enlace a la pediatría integral. Revista Médica IMSS 1995;33:353-359.

34. Packman W, Mazaheri M, Sporri L, Long JK et al. Proyective drawings as measures of psychological functioning in siblings with an intellectual disability: Parental perspectives in two disorders. J Intellectual Disability Research 2008;52:216-229.

35. Wilkens $\mathrm{K}$, Woodgate R. A review of qualitative research on the childhood cancer experience from the perspective of siblings: A need to give them voice. J Pediatric Oncology 2005;22:305-319. 


\section{ANEXO 1. Evaluación de los subsistemas familiares de pacientes pediátricos hospitalizados por enfermedad crónica (eSisFam)}

\begin{tabular}{|c|c|c|c|c|c|}
\hline Sis & ema general & Nunca & A veces & $\begin{array}{c}\text { Casi } \\
\text { siempre }\end{array}$ & Siempre \\
\hline & En esta etapa de la enfermedad, en nuestra familia. & & & & \\
\hline 1 & ...nos mantenemos en contacto para saber cómo va todo & & & & \\
\hline 2 & contamos con el apoyo de nuestros familiares & & & & \\
\hline 3 & mostramos tanto los sentimientos positivos como los negativos & & & & \\
\hline 4 & podemos hablar abiertamente sobre lo que pensamos de la enfermedad & & & & \\
\hline 5 & somos capaces de buscar ayuda en otras personas & & & & \\
\hline 6 & tenemos la capacidad de tomar decisiones que beneficien a la familia & & & & \\
\hline 7 & a pesar de la enfermedad, predomina la armonía & & & & \\
\hline 8 & platicamos sobre lo que sentimos por la enfermedad & & & & \\
\hline 9 & escuchamos nuestros diferentes puntos de vista sobre la enfermedad & & & & \\
\hline 10 & . planeamos actividades para cubrir las necesidades de la familia & & & & \\
\hline 11 & los intereses y necesidades de cada uno también son importantes & & & & \\
\hline 12 & ..cuando alquien se enoja por la situación del paciente, puede expresarlo & & & & \\
\hline 13 & podemos hablar unos con otros acerca de la tristeza que sentimos & & & & \\
\hline 14 & hemos podido organizar nuestras actividades para atender la enfermedad & & & & \\
\hline 15 & ..sabemos cuándo es necesario un cambio en la familia & & & & \\
\hline 16 & podemos expresar la tristeza con llanto & & & & \\
\hline 17 & nos da pena llorar frente a otras personas & & & & \\
\hline 18 & nos ha sido difícil aceptar que esto nos esté pasando & & & & \\
\hline 19 & el cuidador principal se mantiene en contacto con otros familiares & & & & \\
\hline 20 & cada integrante asume la responsabilidad que le corresponde & & & & \\
\hline 21 & .. a pesar de la enfermedad, tenemos tiempo para escucharnos & & & & \\
\hline 22 & . podemos conversar sobre la enfermedad y su tratamiento sin temor & & & & \\
\hline 23 & .es difícil cumplir con las reglas del hospital & & & & \\
\hline 24 & hemos podido organizar el hogar en torno a la enfermedad & & & & \\
\hline 25 & nos demostramos el cariño que nos tenemos & & & & \\
\hline 26 & 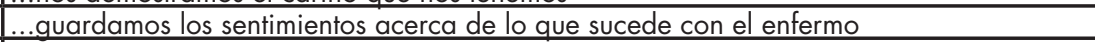 & & & & \\
\hline 27 & las necesidades del cuidador principal también importan a los demás & & & & \\
\hline 28 & .nos cuesta trabajo cambiar nuestros hábitos o rutinas & & & & \\
\hline 29 & pensamos que la responsabilidad del paciente es de todos & & & & \\
\hline 30 &. se permite llorar cuando estamos tristes por el paciente & & & & \\
\hline 31 & hablamos abiertamente de lo que nos está pasando & & & & \\
\hline 32 & creemos que somos capaces de salir adelante con esta difícil situación & & & & \\
\hline 33 & planeamos actividades para cubrir las necesidades del paciente & & & & \\
\hline 34 & cuando tenemos ganas de llorar, lo hacemos & & & & \\
\hline 35 & . platicamos sobre lo que puede suceder con el paciente en el futuro & & & & \\
\hline 36 & peleamos o discutimos por causa de esta situación & & & & \\
\hline 37 & hay unión para enfrentar la enfermedad & & & & \\
\hline 38 & .ocultamos la gravedad de la enfermedad & & & & \\
\hline 39 & hablamos de la enfermedad con nuestros amigos & & & & \\
\hline 40 & .andamos "como en otro planeta" & & & & \\
\hline 41 &. a pesar de la situación difícil, nos damos tiempo para distraernos & & & & \\
\hline 42 & hablamos sobre las necesidades del paciente & & & & \\
\hline 43 &..$e s$ difícil llegar a acuerdos con respecto a la enfermedad & & & & \\
\hline 44 & . se apoya a la persona que más cuida al paciente & & & & \\
\hline 45 & nos ha sido difícil hacer cosas diferentes o nuevas debido a la enfemedad & & & & \\
\hline 46 &.. aunque no estemos presentes, nos mantenemos interesados en la situación del paciente & & & & \\
\hline 47 & ...cuando surge algún problema por la enfermedad, proponemos soluciones & & & & \\
\hline 48 & ...es importante mantenernos informados sobre la enfermedad & & & & \\
\hline 49 & $\begin{array}{l}. . \text { somos organizados para visitar al enfermo en el hospital } \\
\text { nal }\end{array}$ & & & & \\
\hline 50 & tratamos de hacer la vida lo más normal posible & & & & \\
\hline 51 & ...guardamos el enojo que pudiéramos sentir por esta situación & & & & \\
\hline 52 & ...en común acuerdo, cubrimos las necesidades del paciente & & & & \\
\hline 53 & $\begin{array}{l}. . \text { cuando alguno tiene un problema, otros se ocupan de ayudarlo } \\
\text {. }\end{array}$ & & & & \\
\hline 54 & $\begin{array}{l}\ldots \text { nos apoyamos unos a otros mientras el paciente está en el hospital } \\
\text { nom }\end{array}$ & & & & \\
\hline 55 & ....se toman decisiones en conjunto para cosas importantes de la enfermedad & & & & \\
\hline 56 & ...hemos podido modificar nuestras costumbres ante la enfermedad y hospitalización & & & & \\
\hline 57 & ...estamos de acuerdo con el reglamento del hospital & & & & \\
\hline 58 & ...hablamos de la enfermedad con vecinos o conocidos & & & & \\
\hline 59 & $\ldots$ podemos hablar de la enfermedad sin gritar, desesperarnos o $\|$ orar & & & & \\
\hline 60 & ..es importante mantenernos juntos en este momento & & & & \\
\hline 61 & ...algunos integrantes muestran desinterés o lejanía & & & & \\
\hline 62 & ...nos ha sido difícil dejar de hacer cosas que antes & & & & \\
\hline
\end{tabular}

Instrucciones: Seleccione con un click del ratón la respuesta que mejor describa a su familia en esta etapa de la enfermedad. 


\section{ANEXO 1. Evaluación de los subsistemas familiares de pacientes pediátricos hospitalizados por enfermedad crónica (eSisFam) (Continuación)}

\begin{tabular}{|c|c|c|c|c|c|}
\hline \multicolumn{2}{|c|}{ Subsistema conyugal } & \multirow{2}{*}{ Nunca } & \multirow{2}{*}{ A veces } & \multirow[t]{2}{*}{$\begin{array}{c}\text { Casi } \\
\text { siempre }\end{array}$} & \multirow{2}{*}{ Siempre } \\
\hline & En esta etapa de la enfermedad, como pareja. & & & & \\
\hline 1 & ...nos queda tiempo para convivir & & & & \\
\hline 2 & ...nos hemos descuidado por atender al paciente & & & & \\
\hline 3 & nuestra sexualidad es importante & & & & \\
\hline 4 & ...comprendemos lo que siente el otro & & & & \\
\hline 5 & ...tratamos de conservar nuestro espacio íntimo & & & & \\
\hline 6 & la enfermedad nos provocó la separación & & & & \\
\hline 7 & ...evitamos el tema de nuestra sexualidad & & & & \\
\hline 8 & ..podemos disfrutarnos mutuamente en este momento & & & & \\
\hline 9 & ..demostramos el amor que sentimos hacia el otro & & & & \\
\hline 10 & la enfermedad ha afectado nuestra privacidad & & & & \\
\hline 11 & ...nos expresamos cariño & & & & \\
\hline 12 & hemos mostrado interés sexual por otra persona & & & & \\
\hline 13 & nos consolamos uno al otro & & & & \\
\hline 14 & ...podemos tener satisfacción sexual & & & & \\
\hline 15 & ...uno de nosotros se siente desplazado por el hijo enfermo & & & & \\
\hline 16 & ....antes que nosotros está nuestro hijo enfermo & & & & \\
\hline 17 & ...ha habido infidelidad & & & & \\
\hline 18 & tratamos de dedicarnos tiempo uno al otro & & & & \\
\hline 19 & .estamos interesados en nuestra relación sexual & & & & \\
\hline 20 & nos hemos alejado emocionalmente el uno del otro & & & & \\
\hline 21 & ...nos damos un tiempo para salir solos & & & & \\
\hline 22 & ha bajado la calidad de nuestras relaciones sexuales & & & & \\
\hline 23 & hemos estado al punto del divorcio a causa de la enfermedad & & & & \\
\hline 24 & ..podemos sentir pasión el uno por el otro & & & & \\
\hline 25 & ...estamos alejados porque uno está en el hospital y el otro trabajando & & & & \\
\hline 26 & nos motivamos para seguir adelante en esta situación & & & & \\
\hline 27 & creemos que debemos divertirnos en pareja & & & & \\
\hline 28 & ...hemos disminuido nuestras relaciones sexuales & & & & \\
\hline 29 & ....sentimos culpa por disfrutar nuestra relación sexual & & & & \\
\hline 30 & ....uno de los dos dice que la enfermedad es un pretexto para engañar al otro & & & & \\
\hline 31 & mostramos interés sexual por el otro & & & & \\
\hline 32 & ...somos atractivos para el otro & & & & \\
\hline 33 & ...si alquno es infiel piensa que el otro hace lo mismo & & & & \\
\hline 34 & nos ha intereresado otra persona & & & & \\
\hline
\end{tabular}

Instrucciones: Seleccione con un click del ratón la respuesta que mejor describa a los padres (o figuras parentales) como pareja en esta etapa de la enfermedad. 


\section{ANEXO 1. Evaluación de los subsistemas familiares de pacientes pediátricos hospitalizados por enfermedad crónica (eSisFam) (Continuación)}

\begin{tabular}{|c|c|c|c|c|c|}
\hline \multicolumn{2}{|c|}{ Subsistema parental } & \multirow{2}{*}{ Nunca } & \multirow{2}{*}{ A veces } & \multirow[t]{2}{*}{$\begin{array}{c}\text { Casi } \\
\text { siempre }\end{array}$} & \multirow{2}{*}{ Siempre } \\
\hline & En esta etapa de la enfermedad, como padres.. & & & & \\
\hline 1 & ...compartimos la responsabilidad de la enfermedad & & & & \\
\hline 2 & ...sabemos como se sienten los hermanos del paciente & & & & \\
\hline 3 & ...hemos tenido problemas para dormir & & & & \\
\hline 4 & compartimos la responsabilidad de los otros hijos en casa & & & & \\
\hline 5 & ...creemos que las necesidades de nuestros hijos sanos son por ahora secundarias & & & & \\
\hline 6 & ..hemos descuidado nuestra salud por atender al paciente & & & & \\
\hline 7 & sentimos que la responsabilidad del hogar es de los dos & & & & \\
\hline 8 & nos ponemos de acuerdo sobre que hacer con el paciente & & & & \\
\hline 9 & hemos alterado nuestra alimentación & & & & \\
\hline 10 & .tenemos el control de esta situación & & & & \\
\hline 11 & nos ponemos de acuerdo para cuidar a los otros hijos & & & & \\
\hline 12 & ..nos repartimos lo que se requiere para atender la enfermedad & & & & \\
\hline 13 & ...organizamos las actividades del hogar en mutuo acuerdo & & & & \\
\hline 14 & ...nos hemos enfermado con frecuencia & & & & \\
\hline 15 & ...nos apoyamos en las tareas de la casa & & & & \\
\hline 16 & ....mientras uno está en el hospital hay quien atienda a los otros hijos & & & & \\
\hline 17 & .... sólo estamos dedicados al paciente ya que los demás hijos pueden esperar & & & & \\
\hline 18 & ....uno lleva más la carga de la situación que el otro & & & & \\
\hline 19 & ....ambos estamos al tanto del enfermo y su padecimiento & & & & \\
\hline 20 & .satisfacemos las necesidades de los otros hijos & & & & \\
\hline 21 & ...estamos al pendiente de las indicaciones médicas & & & & \\
\hline 22 & ..somos responsables con las necesidades de los otros hijos & & & & \\
\hline 23 & estamos informados de lo que sucede con el enfermo en el hospital & & & & \\
\hline 24 & .nos brindamos apoyo económico & & & & \\
\hline 25 & ...nos brindamos apoyo moral & & & & \\
\hline 26 & ..creemos que los hijos sanos requieren más atención & & & & \\
\hline 27 & .nos apoyamos en las decisiones sobre procedimientos médicos & & & & \\
\hline 28 & ...nos sentimos culpables de la enfermedad de nuestro hijo & & & & \\
\hline 29 & ...nos molesta que nuestros hijos sanos no comprendan esta situación & & & & \\
\hline 30 & ....aunque uno esté ausente del hospital, se mantiene al tanto de la situación & & & & \\
\hline 31 & ....uno le echa la culpa al otro de la enfermedad de nuestro hijo & & & & \\
\hline 32 & ...nos sentimos frustrados ante esta enfermedad & & & & \\
\hline 33 & ....creemos que la atención que ponemos a los otros hijos es adecuada & & & & \\
\hline 34 & ...hemos padecido dolores de cabeza, de estómago, del cuerpo u otro malestar & & & & \\
\hline 35 & ...estamos enojados por esta difícil situación & & & & \\
\hline 36 & sentimos que los hijos sanos exigen más atención de la que les podemos dar & & & & \\
\hline 37 & ...nos sentimos impotentes ante la enfermedad de nuestro hijo & & & & \\
\hline 38 & ..consentimos más a nuestro hijo enfermo que a sus hermanos & & & & \\
\hline 39 & ...estamos desanimados con la situación actual de nuestro hijo & & & & \\
\hline 40 & ..tomamos en cuenta la opinión de los otros hijos & & & & \\
\hline 41 & ...estamos deprimidos & & & & \\
\hline 42 & ...escuchamos a nuestros otros hijos cuando quieren conversar & & & & \\
\hline 43 & ...estamos ansiosos & & & & \\
\hline 44 & ... demostramos cariño a nuestros otros hijos & & & & \\
\hline 45 & ...estamos preocupados & & & & \\
\hline 46 & ....cubrimos las necesidades de los hijos aunque su hermano esté en el hospital & & & & \\
\hline 47 & ...nos encontramos desesperados & & & & \\
\hline 48 & ....uno o ambos ha enfermado a causa de esta situación & & & & \\
\hline 49 & ..hemos tenido que abandonar a los otros hijos por atender al paciente & & & & \\
\hline 50 & ...uno o ambos ya estamos cansados o fatigados por la situación & & & & \\
\hline 51 & ....sabemos lo que sienten nuestros hijos por su hermano enfermo & & & & \\
\hline 52 & cuando nos sentimos enfermos, acudimos a consulta médica & & & & \\
\hline 53 & ...entendemos el estado de ánimo de nuestros hijos sanos & & & & \\
\hline 54 & ....acudimos a nuestro chequeo médico rutinario (dentista, ginecólogo, etc.) & & & & \\
\hline
\end{tabular}

Instrucciones: Seleccione con un click del ratón la respuesta que mejor describa a los padres (o figuras parentales) en su función como padres del paciente en esta etapa de la enfermedad. 


\section{ANEXO 1. Evaluación de los subsistemas familiares de pacientes pediátricos hospitalizados por enfermedad crónica (eSisFam) (Continuación)}

\begin{tabular}{|c|c|c|c|c|c|}
\hline \multicolumn{2}{|c|}{ Subsistema filial } & \multirow[t]{2}{*}{ Nunca } & \multirow[t]{2}{*}{ A veces } & \multirow[t]{2}{*}{$\begin{array}{c}\text { Casi } \\
\text { siempre }\end{array}$} & \multirow[t]{2}{*}{ Siempre } \\
\hline & En esta etapa de la enfermedad, los hermanos del paciente. & & & & \\
\hline 1 & ...están enterados de la enfermedad de su hermano & & & & \\
\hline 2 & .ayudan en los quehaceres del hogar mientras estamos en el hospital & & & & \\
\hline 3 & han tenido cambios bruscos en su estado de ánimo & & & & \\
\hline 4 & 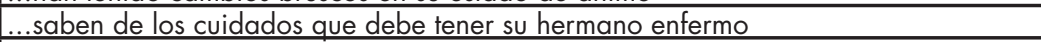 & & & & \\
\hline 5 & .. comprenden que estemos ocupados con su hermano en el hospital & & & & \\
\hline 6 & ...se han enfermado con frecuencia & & & & \\
\hline 7 & .les decimos cómo está su hermano enfermo cuando está en el hospital & & & & \\
\hline 8 & han presentado problemas de conducta & & & & \\
\hline 9 & les disgusta participar en la a atención de su hermano cuando está en casa & & & & \\
\hline 10 & ..juegan o conviven con su hermano enfermo & & & & \\
\hline 11 & . se han mostrado agresivos o rebeldes & & & & \\
\hline 12 & han platicado con el paciente sobre su enfermedad & & & & \\
\hline 13 & han mostrado celos por la atención que damos al paciente & & & & \\
\hline 14 & preguntan como reclamo " ipor qué mi hermano tiene esta enfermedad?" & & & & \\
\hline 15 & han bajado su rendimiento escolar & & & & \\
\hline 16 & Se anqustian por lo que pueda suceder con la vida de su hermano & & & & \\
\hline 17 & han mostrado enojo por la situación de su hermano enfermo & & & & \\
\hline 18 & han padecido dolores de cabeza, de estómago, del cuerpo u otro malestar & & & & \\
\hline 19 & saben en qué consiste esta enfermedad & & & & \\
\hline 20 & han presentado insomnio, pesadillas u otros problemas para dormir & & & & \\
\hline 21 & tienen información clara de la enfermedad & & & & \\
\hline 22 & .se han sentido culpables por la enfermedad de su hermano & & & & \\
\hline 23 & .comprenden la situación actual del paciente & & & & \\
\hline 24 & .se les dificulta quedarse al cuidado de otras personas & & & & \\
\hline 25 & les explicamos en qué consiste el tratamiento a manera que lo entiendan & & & & \\
\hline 26 & envidian el cariño o la atención que demostramos hacia nuestro hijo enfermo & & & & \\
\hline 27 & ..cooperan en las actividades del hogar como cada uno puede & & & & \\
\hline 28 & dicen que a su hermano enfermo, lo consentimos más que a ellos & & & & \\
\hline 29 & . desean visitar a su hermano en el hospital & & & & \\
\hline 30 & tienen miedo de enfermar como su hermano & & & & \\
\hline 31 & tienen información verdadera de la enfermedad & & & & \\
\hline 32 & han mostrado resentimiento hacia su hermano enfermo & & & & \\
\hline 33 & ..parecen entender cuando les explicamos esta situación & & & & \\
\hline 34 & .han expresado sentir soledad por la ausencia de su hermano & & & & \\
\hline 35 & les explicamos algunos procedimientos médicos de manera que los entiendan & & & & \\
\hline 36 & se han mostrado desanimados & & & & \\
\hline 37 & han presentado problemas en su alimentación & & & & \\
\hline 38 & han desarrollado miedos & & & & \\
\hline 39 & le hablan por teléfono o le envían una carta al paciente cuando está en el hospital & & & & \\
\hline 40 & .se han sentido solos porque los padres están en el hospital & & & & \\
\hline 41 & se muestran comprensivos con el paciente & & & & \\
\hline 42 & muestran rechazo por su hermano enfermo & & & & \\
\hline 43 & participan en el cuidado de su hermano enfermo cuando está en casa & & & & \\
\hline 44 & ..son intolerantes con su hermano enfermo & & & & \\
\hline 45 & ..se avergüenzan de su hermano enfermo & & & & \\
\hline 46 & .se han aislado de los demás & & & & \\
\hline 47 & han mostrado aceptación por el hermano enfermo & & & & \\
\hline 48 & expresan sentirse enfermos igual que el paciente & & & & \\
\hline 49 & ..cuidan a su hermano enfermo de caídas, golpes o algo que pudiera lastimarlo & & & & \\
\hline 50 & .se han mostrado angustiados & & & & \\
\hline 51 & animan al paciente a cuidar su salud & & & & \\
\hline 52 & ..han visto afectadas algunas de sus actividades a causa de la enfermedad & & & & \\
\hline 53 & muestran cariño hacia su hermano enfermo & & & & \\
\hline 54 & han expresado sentir culpa por estar sanos & & & & \\
\hline 55 & .. debido a la gravedad de la enfermedad, les hemos tenido que mentir & & & & \\
\hline 56 & tratan a su hermano enfermo como cualquier persona normal & & & & \\
\hline 57 & se molestan por la atención que le ponemos a su hermano enfermo & & & & \\
\hline 58 & han tratado de chantajear o manipular aprovechándose de esta situación & & & & \\
\hline 59 & están tranquilos ante esta situación & & & & \\
\hline
\end{tabular}

